\title{
Escala de Comunicación Familiar: validación en población adulta chilena
}

\author{
Joucelyn Rivadeneira* y Miguel Angel López \\ Universidad del Bío-Bío, Chile
}

Recibido, octubre 21/2016

Concepto de evaluación, febrero 15/2017

Aceptado, marzo 15/2017
Referencia: Rivadeneira, J. \& López, M. A. (2017). Escala de comunicación familiar: validación en población adulta chilena. Acta Colombiana de Psicología. 20(2), 116-126. doi: http://www.dx.doi.org/10.14718/ACP.2017.20.2.6

\section{Resumen}

Es a través de la comunicación que se producen las más tempranas socializaciones del ser humano, proceso mediante el cual se construye la familia y la sociedad. El objetivo de este estudio fue analizar las propiedades psicométricas de la versión española de la Escala de Comunicación Familiar, en una muestra aleatoria de 340 adultos chilenos. Se aplicó el análisis factorial exploratorio (ejes principales) y confirmatorio (libre distribución asintótica) ajustado a datos sin distribución normal multivariante (test de Doornik-Hansen de $\mathrm{p}<.001$ ). Se encontró una solución factorial de dos constructos con prueba de $\chi 2=9.466(\mathrm{p}=.305), \mathrm{CFI}=.983, \mathrm{TLI}=.968, \mathrm{RMSEA}=.023(\mathrm{p}=.781)$ y SRMR $=.104$, además de consistencias internas de .895 y .854 para cada constructo. La evidencia obtenida en este tipo de sujetos apoya la idea de que la escala mide adecuadamente la variable de interés por medio de dos constructos y seis reactivos. Los datos confirman los buenos indicadores psicométricos que apoyan el uso de la escala en el área de la investigación y la intervención familiar.

Palabras clave: Comunicación, familia, escala, propiedades psicométricas, validación.

\section{Family Communication Scale: Validation in Chilean adult population}

\begin{abstract}
The earliest socialization in human beings, a process whereby family and society are built, takes place through communication. The aim of this study was to analyze the psychometric properties of the Spanish version of the Family Communication Scale using a random sample of 340 Chilean adults. Exploratory factor analysis (principal axis) and confirmatory factor analysis (free asymptotic distribution) adjusted to data without multivariate normal distribution were applied (Doornik-Hansen test: $\mathrm{p}<.001)$. A factorial solution of two constructs was found with $\chi 2$ test=9.466 ( $\mathrm{p}=.305), \mathrm{CFI}=.983, \mathrm{TLI}=.968, \mathrm{RMSEA}=.023$ $(\mathrm{p}=.781)$, and $\mathrm{SRMR}=.104$, as well as internal consistencies of .895 and .854 in both constructs. The evidence obtained supports the idea that the scale adequately measures the variable of interest through two constructs and six items. The data confirm the good psychometric indicators that support the use of the scale in the area of research and family intervention. Key words: communication, family, scale, psychometric properties, validation.
\end{abstract}

* Departamento Ciencias Sociales, Avenida Brasil 1180 Chillán (Chile),+56(42)2463418, jrivadeneira@ubiobio.cl

** Departamento de Ciencias de la Salud, Avenida Andrés Bello 720 Chillán (Chile), miglopeze@gmail.com 


\title{
Escala de Comunicaçáo Familiar: validaçáo em populaçáo adulta chilena
}

\begin{abstract}
Resumo
A comunicação é o processo pelo qual são produzidas as mais precoces socializações do ser humano, além de se construir a família e a sociedade. O objetivo deste estudo foi analisar as propriedades psicométricas da versão espanhola da Escala de Comunicação Familiar numa amostra aleatória de 340 adultos chilenos. Aplicou-se a análise fatorial exploratória (eixos principais) e confirmatória (livre distribuição assintótica) ajustada a dados sem distribuição normal multivariante (teste de Doornik-Hansen de $\mathrm{p}<, 001)$. Encontrou-se uma solução fatorial de dois construtos com teste de $\chi 2=9,466(\mathrm{p}=, 305)$, $\mathrm{CFI}=, 983, \mathrm{TLI}=, 968, \mathrm{RMSEA}=, 023(\mathrm{p}=, 781)$ e SRMR $=, 104$, além de consistências internas de $, 895 \mathrm{e}, 854$ para cada construto. A evidência obtida nesse tipo de sujeitos apoia a ideia de que a escala mede adequadamente a variável de interesse por meio de dois construtos e seis reativos. Os dados confirmam os bons indicadores psicométricos que apoiam o uso da escala na área da pesquisa e da intervenção familiar.

Palavras-chave: comunicação, escala, família, propriedades psicométricas, validação.
\end{abstract}

\section{INTRODUCCIÓN}

El interés académico por la comunicación familiar se remonta a la década de los 40, sin embargo, es en la década de los 70 cuando, a partir de las contribuciones derivadas de diversas investigaciones, comienza a surgir una evolución en este campo (Galvin, 2015; Webb \& Dickson, 2012).

La comunicación familiar es entendida, según Tesson y Youniss (1995), como el instrumento que padres e hijos utilizan para renegociar sus roles, desarrollar sus relaciones y cambiar hacia una mayor mutualidad y reciprocidad. Precisamente, es a través del proceso de socialización llevado a cabo al interior de la familia que los hijos adquieren los componentes culturales y parámetros sociales que facilitan su integración social (Musitu \& Cava, 2001). En este sentido, la comunicación familiar forma parte del contexto familiar, elemento reconocido por su importancia para el desarrollo infantil debido a las prácticas formativas que se llevan a cabo a través de la estructura y dinámica que facilitan las experiencias de aprendizaje para el desempeño social (Isaza \& Henao, 2011).

En efecto, se reconoce a la familia como el principal contexto de desarrollo en el que se producen los aprendizajes más significativos para el ser humano (Bronfenbrenner, 2005), y, en su interior, resalta la importancia de la comunicación, foco obligado cuando se intentan explicar algunos fenómenos sociales complejos como, por ejemplo, la violencia familiar (Corsi, 1999), el abuso sexual (Perrone \& Nannini, 2000) o el maltrato infantil (Barcelata \& Álvarez, 2005). De esta forma, la evaluación de la comunicación familiar permite comprender distintos factores asociados no solo al funcionamiento de la familia, sino a elementos del clima familiar que pueden contribuir a explicar las trayectorias evolutivas emocionales de sus miembros, lo que implica que se trata de una herramienta vital en los procesos biopsicosociales de intervención.

\section{La comunicación y el desarrollo humano en la familia}

Es de amplio consenso considerar a la familia como el eje de la sociedad y como la institución donde se desarrollan los principales aprendizajes del ser humano. La familia basa su funcionamiento en la interacción y los significados que sus miembros le otorgan a los procesos comunicativos (Gallego, 2006); y es a partir de estos que construyen su realidad familiar, sobre todo a través de la conversación y la co-creación de la vida privada y de las visiones que se puedan tener del mundo; lo que, además, da paso a patrones comunicativos donde se confrontan la necesidad de expresión individual frente a la privacidad comunicativa.

Estos patrones o modelos de comunicación familiar varían a lo largo del ciclo de vida familiar, aunque, a pesar de los cambios, mantienen una tendencia estable y predecible (Koerner \& Fitzpatrick, 2004). Así mismo, la comunicación familiar es reconocida como un proceso dinámico y bidireccional en el sentido de que padres e hijos se influyen mutuamente, y que, según Banovcinova y Levicka (2015), puede estar influenciada por dos factores: a) las características de la familia, los individuos y el contexto social, donde se incluyen el ambiente familiar, las redes de interacción, las normas, el contexto sociocultural y los patrones comunicacionales; $\mathrm{y} b$ ) las características del proceso de comunicación en sí, como la modalidad comunicativa —oral o escrita, por ejemploel nivel y las formas de comunicación.

Dentro del primer grupo destaca el trabajo de Fitzpatrick y Ritchie (1993) quienes en un intento por describir la influencia mutua entre la comunicación familiar y el ambiente familiar crearon dos conceptos que han sido fundamentales en estudios posteriores: la orientación a la 
conformidad y la orientación a la conversación. Por una parte, la orientación a la conversación se entiende como el grado en el que las familias crean un clima que anima a sus miembros a opinar y compartir pensamientos, sentimientos y actividades; mientras que la orientación a la conformidad se refiere al grado en el que la comunicación familiar propicia la homogeneidad en actitudes, valores y creencias. Ambas dimensiones han facilitado la comprensión y el estudio de aspectos funcionales de la familia en relación a la comunicación.

\section{El estudio de la comunicación familiar}

La evaluación de la comunicación familiar ha avanzado de manera importante en las últimas décadas, y el conocimiento científico en torno a la repercusión que determinadas características pueden tener en el desarrollo humano se ha expandido considerablemente.

Existe evidencia de que una familia con comunicación positiva - es decir, que genera mensajes claros y congruentes, provee soporte, demuestra afecto, y afronta con habilidades la resolución de conflictos - es capaz de afrontar de mejor manera los desafíos de la educación en el hogar; mientras que una familia con comunicación negativa - que utiliza frecuentemente la crítica, la negación de los sentimientos, el excesivo conflicto, y que no sabe escuchar - tiene menor capacidad de afrontar de manera adecuada la educación de los hijos (Segrin \& Flora, 2011; Smith, Freeman \& Zabriskie, 2009).

En esta misma línea, los hallazgos indican que la comunicación familiar abierta y fluida se asocia con una serie de resultados positivos en el ámbito familiar e individual. En concreto, la comunicación familiar positiva tiene un efecto de protección ante las conductas delictivas en los adolescentes (Jiménez, Murgui, Estévez \& Musitu, 2007; Kerr \& Stattin, 2000), en la resolución de conflictos (Girbau, 2002; Pérez \& Aguilar, 2009) y en el ajuste escolar (Estévez, Musitu \& Herrero, 2005; Martínez, Musitu, Murgui \& Amador, 2009).

Sin embargo, existe menor consenso en relación a los resultados en torno a una comunicación familiar menos fluida: en algunos estudios se señala que esta puede constituir un factor de riesgo en el desarrollo emocional de los hijos (Estévez, Herrera, Martínez \& Musitu, 2006; Lambert \& Cashwell, 2003), mientras que otras investigaciones han demostrado que ante la presencia de una comunicación familiar escasa, los conflictos entre padres e hijos adolescentes también son menores, presumiblemente debido a la evitación de temas relacionados con ámbitos personales en el desarrollo y futuro de los adolescentes (Luna, 2012; Parra \& Oliva, 2002).
Asimismo, la investigación actual en torno a la comunicación familiar se ha diversificado a otras áreas multidisciplinarias como ocurre en la salud (Kodali et al., 2015; Leenen et al., 2016; Myers, Fernandes, Arduser, Hopper \& Koehly, 2015; O'Toole et al., 2015), la satisfacción familiar (Burns \& Pearson, 2011; Levin, Dallago \& Currie, 2012), y las nuevas tecnologías (Rudy, Dworkin, Walker \& Doty, 2015; Wang et al., 2015).

Con respecto a las investigaciones internacionales sobre la comunicación familiar, estas tienen una fuerte raíz en Europa y Estados Unidos. Como consecuencia de ello, los resultados obtenidos distan de la realidad social y cultural de América Latina. En particular, la situación en Chile muestra que han habido avances significativos en la validación de instrumentos orientados a ámbitos relacionados con la familia, así como en el desarrollo de la infancia y la adolescencia (Lecannelier et al., 2014; Mathiesen, Merino, Herrera, Castro \& Rodríguez, 2011; Quintana \& Muñoz, 2010; Saldivia, Vicente, Valdivia \& Melipillán, 2013), el contexto escolar (Lecannelier et al., 2011; López, Bilbao, Ascorra, Moya \& Morales, 2014) y aspectos generales del funcionamiento familiar (Puschel, Repetto, Solar, Soto \& Gonzalez, 2012; Retamales, Behn \& Merino, 2004; Zicavo, Palma \& Garrido, 2012); sin embargo, los estudios que han abordado específicamente la comunicación familiar son prácticamente escasos (Santander et al., 2008). Razón por la cual se aprecia un vacío empírico al respecto y una carencia de instrumentos que permitan evaluar esta característica familiar en el contexto nacional.

Considerando la importancia de contar con un instrumento fiable para la medición de la comunicación familiar en Chile, en el presente estudio se planteó como objetivo desarrollar una versión para la población chilena de la adaptación española de la Escala de Comunicación Familiar (Sanz, Iraurgi \& Martínez-Pampliega, 2002). Esta escala ha sido aplicada en otros estudios internacionales (Presa, 2015; Rivadeneira, 2013; Rivero \& Martínez-Pampliega, 2010) y tiene una evidencia preliminar que sugiere que puede tratarse de una herramienta útil para la medición de la comunicación familiar.

\section{MÉTODO}

\section{Diseño}

La presente investigación se enmarca en el enfoque de construcción de conocimiento empírico-analítico; y, específicamente, se trata de un estudio descriptivo-explicativo 
sobre las propiedades psicométricas y la validación estructural de la Escala de Comunicación Familiar.

\section{Participantes}

La población del estudio tuvo como base el registro total de estudiantes escolarizados entre el cuarto y el sexto año de educación básica de la ciudad de Chillán, en Chile, durante el año 2015. De acuerdo a los datos estadísticos del Departamento Provincial de Educación, en ese año el total de estudiantes ascendía a 5124, los cuales se repartían en 33 establecimientos educativos municipales y 35 establecimientos educativos en modalidad subvencionadaparticular. Para obtener la muestra del estudio, se escogieron de manera aleatoria simple tres establecimientos educacionales por unidad administrativa; posteriormente, se realizó un muestreo por conglomerados, teniendo en cuenta los niveles cuarto, quinto y sexto básico. De esta forma, la muestra quedó compuesta por 340 madres, padres o adultos responsables de los jóvenes de esos niveles. Este tamaño de muestra satisface una solución factorial confirmatoria unidimensional o bidimensional, con un poder de $1-\beta=.83$ y un peso factorial mínimo de $\lambda=.50$ según la propuesta de Wolf, Harrington, Clark y Miller (2013).

Los datos descriptivos principales de la población indican que la edad promedio de los adultos fue de 40.14 años $(\mathrm{DT}=10.18)$. Del total de participantes, el $83.8 \%$ eran madres, $13.8 \%$ padres y $2.4 \%$ otros adultos responsables. Con respecto a su nivel educativo, $19.1 \%$ reportaron estudios básicos, $50.9 \%$ estudios de enseñanza media y $28.2 \%$ nivel de estudios universitarios y post-universitarios. Y, por otro lado, $55.1 \%$ se encontraba en una situación laboral activa o remunerada (véase Tabla 1).

\section{Instrumento}

Se utilizó la Escala de Comunicación Familiar - Family Comunication Scale (FCS) — elaborada por Barnes y

Tabla 1

Distribución de los participantes del estudio según sus principales características sociodemográficas

\begin{tabular}{|c|c|c|c|}
\hline Características & Media (dt) & Frecuencia & $(\%)$ \\
\hline Edad & $40.14(10.18)$ & & \\
\hline \multicolumn{4}{|l|}{ Tipo de adulto } \\
\hline Madre & & 285 & $(83.8)$ \\
\hline Padre & & 47 & $(13.8)$ \\
\hline Otro adulto responsable & & 8 & $(2.4)$ \\
\hline \multicolumn{4}{|l|}{ Estado civil } \\
\hline Soltero/a & & 89 & $(26.2)$ \\
\hline Casado/a & & 190 & $(55.9)$ \\
\hline Con pareja estable & & 39 & (11.5) \\
\hline Viudo/a & & 5 & $(1.5)$ \\
\hline Separado/a & & 16 & $(4.7)$ \\
\hline No contesta & & 1 & $(.3)$ \\
\hline \multicolumn{4}{|l|}{ Nivel de estudios } \\
\hline Básica & & 65 & $(19.1)$ \\
\hline Media & & 173 & $(50.9)$ \\
\hline Universitaria & & 90 & $(22.9)$ \\
\hline Post grado & & 6 & (1.8) \\
\hline No contesta & & 6 & $(1.8)$ \\
\hline \multicolumn{4}{|l|}{ Situación laboral } \\
\hline Trabajador activo & & 190 & $(55.9)$ \\
\hline Sin trabajo & & 96 & $(28.2)$ \\
\hline Jubilado (pensionado) & & 6 & $(1.8)$ \\
\hline No realiza trabajo remunerado & & 40 & $(11.8)$ \\
\hline No contesta & & 8 & $(2.4)$ \\
\hline
\end{tabular}


Olson (1982), adaptada y validada en España por Sanz et al. (2002). El instrumento, en su versión española, muestra un coeficiente de consistencia interna - alfa de Cronbachde .88 y una correlación test-retest e intraclase de .88. En cuanto a la validez concurrente, esta escala se correlacionó con constructos teóricos afines de la Escala de Clima Social en la Familia (Moos, Moos \& Trickett, 1987), en donde se encontró una correlación positiva con las dimensiones de comunicación (.68) y expresividad (.59), y una correlación negativa con la dimensión de conflicto (-.37). Estas asociaciones indican la existencia de validez convergente y divergente de la escala en su versión española (Sanz et al., 2002).

En particular, el objetivo de esta escala es evaluar la comunicación que se produce en la familia, recogiendo aspectos importantes como el nivel de apertura o libertad para intercambiar ideas, la información y preocupaciones entre generaciones, la confianza y honestidad experimentada, y el tono emocional de las interacciones. Se trata de un instrumento que por su brevedad y sencillez permite la aplicación individual y grupal. Consta de 10 ítems en una escala unidimensional que permiten valorar las habilidades positivas de comunicación, como lo son los mensajes claros y congruentes, la empatía, las frases de apoyo y las habilidades efectivas de resolución de problemas. Por otro lado, el tiempo de aplicación es de aproximadamente diez minutos; y para la aplicación, cada ítem se puntúa sobre una escala con cinco alternativas de respuesta: (1) no describe nada a mi familia; (2) solo la describe ligeramente; (3) describe a veces a mi familia; (4) en general, sí describe a mi familia; y (5) describe muy bien a mi familia.

La puntuación total se obtiene de la suma de las puntaciones; así, la puntuación mínima es de 10 puntos y la máxima de 50 puntos; $y$, para la calificación, entre más alta sea la puntuación, mejor nivel de comunicación familiar.

En el presente estudio, cada uno de los 10 ítems fue sometido a un análisis de pertinencia lingüística por parte de tres jueces independientes $y$, teniendo en cuenta sus aportes, no se efectuaron cambios a la escala.

\section{Procedimiento}

Tanto las familias como los directores de los establecimientos educativos recibieron información sobre los objetivos del estudio y se le solicitó la firma del consentimiento informado a las familias para participar voluntariamente en el estudio. Previo a la aplicación del instrumento, los encuestadores se sometieron a un entrenamiento supervisado con el objetivo de uniformar el método de recogida de los datos, el cual incluyó la forma de administrar el instrumento. La aplicación se llevó a cabo de forma colectiva en las reuniones de curso programadas por los establecimientos educativos.

\section{RESULTADOS}

Como resultado se describe la conformación de los constructos del instrumento con el análisis factorial exploratorio según el método de ejes principales, puesto que los datos no presentaron normalidad multivariante -medida con el test de Doornik-Hansen- $(\mathrm{Chi} 2=1543.012 ; 20 \mathrm{gl}$; $\mathrm{p}<.001)$. Se rotaron los datos con el método Varimax para facilitar la definición de los constructos, minimizando el número de reactivos que tienen saturaciones altas en cada factor; y para evaluar la factibilidad de ejecutar el análisis factorial exploratorio se aplicó la prueba de esfericidad de Bartlett y el coeficiente de Kaiser-Meyer-Olkin (KMO). Los constructos extraídos obedecieron a la aplicación de tres criterios: a) autovalor superior a 1.00 ; b) método gráfico codo; y c) varianza explicada ( $60 \%$ o más). Y para la solución factorial se debían incluir al menos tres ítems por constructo.

Luego de conocer la cantidad de constructos que contiene el instrumento, se aplicó el análisis factorial confirmatorio para evaluar si los ítems correlacionaban adecuadamente con los constructos, además del nivel de relación entre dichos constructos, la magnitud de los errores de medida y el ajuste global del modelo especificado a los datos muestrales. Posteriormente, se empleó el modelo de ecuaciones estructurales con el método de libre distribución asintótica. Todas las estimaciones se muestran como valores estandarizados.

Existe evidencia de validez por análisis factorial confirmatorio cuando se acepta la hipótesis nula que plantea que la diferencia entre la matriz de varianza-covarianza observada en la muestra y la estimada por el modelo estructural es igual a cero. El nivel de significación de la escala en este estudio fue de .05 . Y dado que esta prueba de hipótesis se basa en el tamaño muestral, también son pruebas de validez confirmatoria los siguientes indicadores de validez: la raíz del residuo cuadrático medio RMSR $<.08$, la raíz del error cuadrático medio de aproximación RMRSEA $<.08$, el índice de ajuste no normalizado NNFI/TLI > .95, y el índice de ajuste comparativo CFI $>.95$ (Arias, 2008; Hooper, Coughlan \& Mullen, 2008; Ruiz, Pardo \& San Martín, 2010; Stage, King, Nora \& Barlow, 2006).

En la Tabla 2 se presentan las puntuaciones medias y la variabilidad de cada ítem que integra la escala aplicada a la muestra de adultos chilenos. En esta, se muestra además que las correlaciones entre el puntaje del ítem con el total de la escala fueron superiores a .60, excepto para el ítem CF_5, que presentó un valor de .57. Al eliminar ese ítem, el valor alfa de Cronbach de la escala muestra valores superiores a .90. Por último, el coeficiente de adecuación muestral por ítem (MSA, por su sigla del inglés), el cual determina el 
nivel de correlación del ítem a un determinado constructo, muestra valores superiores a .90 .

Para evaluar la factibilidad empírica de construir un análisis factorial exploratorio se obtuvo una prueba de esfericidad de Bartlett estadísticamente significativa, donde se obtuvieron resultados de $\left(\chi^{2}=1980.786 ; 45 \mathrm{gl} ; \mathrm{p}<.001\right)$, además del coeficiente $\mathrm{KMO}=.93$, que serían pruebas que apoyan la presencia de constructos para analizar.

Se aplicó un análisis factorial para un constructo, dado que la versión española de la escala que se utilizó para generar este estudio se presenta como unidimensional (Sanz et al., 2002). En este, se encontró que con la muestra escogida se logra explicar el $54.2 \%$ de la varianza, con comunalidades superiores a .50 , excepto para los ítems CF_6 (.495) y CF_5 (.354). El alfa de Cronbach de los 10 ítems de la escala fue de .919 (véase Tabla 3, Solución A).

Posteriormente, se generó una segunda solución factorial, esta vez con dos constructos (Tabla 3, Solución B), en donde se encontró que estos dos suman el $60.1 \%$ de la varianza, con un único reactivo con valor de comunidad inferior a $.50(\mathrm{CF} 5)$ y con pesos factoriales que se diferencian entre los dos constructos (a excepción de CF_8 y CF_7), sin embargo, en ambos constructos se encontraron valores alfa superiores a .80. Por otro lado, se generó una tercera solución factorial (no publicada), la cual explicó un $60.01 \%$ de la varianza, en donde el tercer factor estuvo compuesto solo por el CF_6, razón por la cual se desechó la posibilidad de presentarlo como una opción factorial para testearlo con análisis factorial confirmatorio. En conclusión, se testeó una solución factorial confirmatoria con dos constructos, empezando por 10 reactivos, con sospecha de que los reactivos CF_5,CF_6, CF_7 y CF_8 no contribuyeran a una solución con buena bondad de ajuste.

En la Tabla 4 se presentan las bondades de ajuste de los modelos de ecuaciones estructurales a partir de esas dos soluciones factoriales exploratorias. En general, se encontró que la solución factorial con dos factores y seis reactivos presenta el mejor ajuste (se eliminó uno en uno)

Tabla 2

Medidas de resumen, correlación ítem-total, coeficiente alfa si se elimina el ítem y coeficiente MSA de todos los items de la escala

\begin{tabular}{|c|c|c|c|c|c|}
\hline Ítems & Media & $\begin{array}{l}\text { Desviación } \\
\text { típica }\end{array}$ & $\mathrm{R}$ ítem-total & $\begin{array}{l}\alpha \text { si se elimina } \\
\text { el ítem }\end{array}$ & $\mathrm{MSA}^{*}$ \\
\hline $\begin{array}{l}\text { CF_1. Los miembros de mi familia estamos } \\
\text { satisfechos con la forma de comunicarnos. }\end{array}$ & 4.25 & .81 & .69 & .91 & .91 \\
\hline $\begin{array}{c}\text { CF_2. Los miembros de mi familia sabemos } \\
\text { escuchar. }\end{array}$ & 4.17 & .83 & .68 & .91 & .91 \\
\hline $\begin{array}{l}\text { CF_3. Los miembros de mi familia nos ex- } \\
\text { presamos afecto entre nosotros. }\end{array}$ & 4.53 & .76 & .74 & .91 & .92 \\
\hline $\begin{array}{l}\text { CF_4. En nuestra familia compartimos los } \\
\text { sentimientos abiertamente. }\end{array}$ & 4.40 & .88 & .77 & .91 & .94 \\
\hline CF_5. Disfrutamos pasando tiempo juntos. & 4.55 & .87 & .57 & .92 & .96 \\
\hline $\begin{array}{l}\text { CF_6. Los miembros de mi familia discutimos } \\
\text { los sentimientos e ideas entre nosotros. }\end{array}$ & 4.24 & .91 & .67 & .91 & .93 \\
\hline $\begin{array}{l}\mathrm{CF}_{\text {_7 }} \text {. Cuando los miembros de mi familia pre- } \\
\text { guntamos algo, las respuestas son sinceras. }\end{array}$ & 4.49 & .75 & .68 & .91 & .96 \\
\hline $\begin{array}{l}\text { CF_8. Los miembros de mi familia intentamos } \\
\text { comprender los sentimientos de los otros. }\end{array}$ & 4.45 & .78 & .69 & .91 & .97 \\
\hline $\begin{array}{l}\text { CF_9. Los miembros de mi familia resolvemos } \\
\text { tranquilamente los problemas. }\end{array}$ & 4.19 & .84 & .72 & .91 & .93 \\
\hline $\begin{array}{c}\text { CF_10. En nuestra familia expresamos nuestros } \\
\text { verdaderos sentimientos. }\end{array}$ & 4.58 & .73 & .78 & .91 & .92 \\
\hline
\end{tabular}

Nota. * Measure of Sampling Adequacy. 
Tabla 3

Solución factorial exploratoria con ejes principales (rotación varimax) de los reactivos que conforman la escala en una muestra de adultos $(n=340)$

\begin{tabular}{|c|c|c|c|c|c|}
\hline \multirow{2}{*}{ Ítems } & \multicolumn{2}{|c|}{ Solución A } & \multicolumn{3}{|c|}{ Solución B } \\
\hline & Factor 1 & Comunalidad & Factor 1 & Factor 2 & Comunalidad \\
\hline $\begin{array}{l}\text { CF_1. Los miembros de mi familia } \\
\text { estamos satisfechos con la forma de } \\
\text { comunicarnos. }\end{array}$ & .724 & .525 & .350 & .737 & .666 \\
\hline $\begin{array}{l}\text { CF_2. Los miembros de mi familia } \\
\text { sabemos escuchar. }\end{array}$ & .715 & .512 & .307 & .788 & .714 \\
\hline $\begin{array}{l}\text { CF_3. Los miembros de mi familia } \\
\text { nos expresamos afecto entre nosotros. }\end{array}$ & .776 & .601 & .733 & .335 & .649 \\
\hline $\begin{array}{l}\text { CF_4. En nuestra familia compartimos } \\
\text { los sentimientos abiertamente. }\end{array}$ & .811 & .658 & .725 & .394 & .682 \\
\hline $\begin{array}{l}\text { CF_5. Disfrutamos pasando tiempo } \\
\text { juntos. }\end{array}$ & .595 & .354 & .583 & & .393 \\
\hline $\begin{array}{l}\text { CF_6. Los miembros de mi familia } \\
\text { discutimos los sentimientos e ideas } \\
\text { entre nosotros. }\end{array}$ & .703 & .495 & .625 & .344 & .509 \\
\hline $\begin{array}{l}\mathrm{CF} \text { 7. Cuando los miembros de mi fa- } \\
\text { milia preguntamos algo, las respuestas } \\
\text { son sinceras. }\end{array}$ & .715 & .511 & .575 & .420 & .507 \\
\hline $\begin{array}{l}\text { CF_8. Los miembros de mi familia } \\
\text { intentamos comprender los sentimien- } \\
\text { tos de los otros. }\end{array}$ & .748 & .559 & .430 & .662 & .622 \\
\hline $\begin{array}{l}\text { CF 9. Los miembros de mi familia } \\
\text { resolvemos tranquilamente los prob- } \\
\text { lemas. }\end{array}$ & .723 & .523 & .553 & .458 & .516 \\
\hline $\begin{array}{l}\text { CF_10. En nuestra familia expresamos } \\
\text { nuestros verdaderos sentimientos. }\end{array}$ & .824 & .678 & .790 & .345 & .743 \\
\hline Autovalor & 5.417 & & 3.455 & 2.546 & \\
\hline Porcentaje de varianza & $54.2 \%$ & & $34.6 \%$ & $25.5 \%$ & \\
\hline Alfa de Cronbach & .919 & & .895 & .854 & \\
\hline
\end{tabular}

Tabla 4

Indicadores de bondad de ajuste de los dos modelos calculados

\begin{tabular}{|c|c|c|c|c|c|}
\hline \multirow{2}{*}{ Modelos } & \multicolumn{5}{|c|}{ Indicador de ajuste } \\
\hline & $\chi^{2 *}(\mathrm{p}>.05)$ & CFI $(>.95)$ & TLI $(>.95)$ & $\operatorname{RMSEA}(<.06)$ & SRMR $(<.08)$ \\
\hline $\begin{array}{l}\text { Con un factor y } 10 \\
\text { reactivos. }\end{array}$ & $\begin{array}{c}98.334 \\
\mathrm{p}<.001\end{array}$ & .438 & .277 & $\begin{array}{c}.073 \\
(\text { IC } 90 \%: .056-.090) \\
p<.014\end{array}$ & .344 \\
\hline $\begin{array}{c}\text { Con dos factores y } 6 \\
\text { reactivos. }\end{array}$ & $\begin{array}{c}9.466 \\
\mathrm{P}=.305\end{array}$ & .983 & .968 & $\begin{array}{c}.023 \\
(\text { IC } 90 \%: .000-.070) \\
p=.781\end{array}$ & .104 \\
\hline
\end{tabular}

Nota. *Bondad de ajuste que evalúa la hipótesis nula de que la diferencia entre la matriz de varianza-covarianza observada en la muestra y la estimada por el modelo estructural es igual a cero. Se espera probar $\mathrm{H}_{0}$. CFI: Índice de bondad de ajuste. TLI: Índice de ajuste no normalizado. RMSEA: Raíz del error cuadrático medio de aproximación. RMSR: Raíz del residuo cuadrático medio. 
en comparación con los resultados con base en la soluciones unifactorial y bifactorial con 10 reactivos.

La Figura 1 presenta los pesos factoriales de las ecuaciones estructurales obtenidas para la versión con dos factores y seis reactivos con la mejor bondad de ajuste. Se encontraron valores superiores a .75 con errores tipificados cercanos a cero, con puntuaciones z superiores a $1.96(\mathrm{p}<.001)$, y una covarianza entre ambos constructos de .80 .

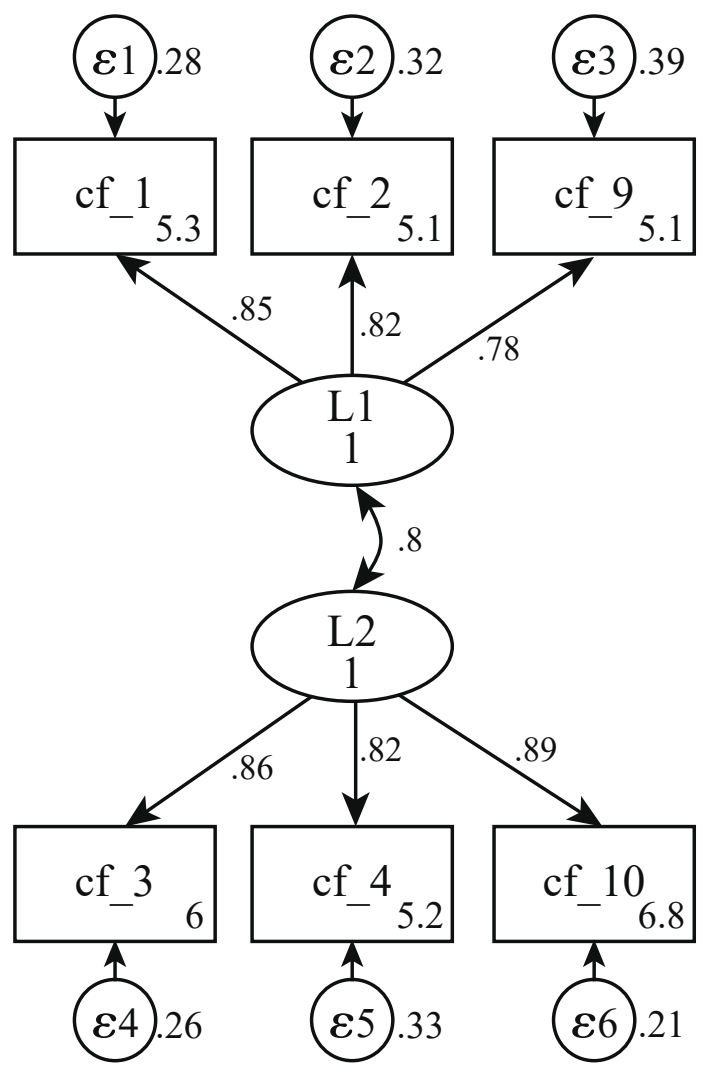

Figura 1. Modelo factorial confirmatorio con el mejor ajuste encontrado.

\section{DISCUSIÓN}

El objetivo de este estudio fue validar la versión española de la Escala de Comunicación Familiar en población chilena. Se encontró que una solución factorial con dos factores y seis reactivos presenta el mejor ajuste, lo que sugiere que este instrumento permite diferenciar entre los componentes emocionales y afectivos de la comunicación, y los componentes asociados a aspectos más generales de la comunicación familiar - como la resolución de conflictos o la capacidad de escucha- en la muestra chilena.

Esta evidencia marca una diferencia con la escala original, en la que se apoya una solución unidimensional. En este sentido, los resultados de esta investigación concuerdan con los supuestos teóricos de Banovcinova y Levicka (2015) mediante los cuales la comunicación se ve influenciada precisamente por estos dos elementos diferenciados: las características de la familia — clima familiar-y las características del proceso de comunicación. En efecto, en esta adaptación del instrumento se aprecia una agrupación de los constructos asociados a la categoría de orientación a la conversación que destacan del trabajo de Koerner y Fitzpatrick (2002), donde se concentran las preguntas dirigidas a determinar el grado en que las familias crean un clima que anima a sus miembros a participar con libertad en las interacciones.

Esto implica que en la población chilena se entienden de manera diferenciada las preguntas que pueden relacionarse con la expresión de sentimientos y emociones ("los miembros de la familia nos expresamos afecto entre nosotros"), de aquellos que apuntan a elementos más generales de la comunicación familiar, como la resolución de conflictos o la capacidad de escuchar ("los miembros de la familia sabemos escuchar").

Sin duda, esta diferencia en el instrumento respecto a la versión española podría explicarse por las características culturales entre ambas poblaciones. La comunicación familiar es un proceso en el cual el contexto es un elemento importante que debe ser considerado; y, puesto que la comunicación familiar se entiende dentro de dicho sistema socialmente aprendido y comprendido, se deben incluir aspectos históricos, sociales y físico (Gallego, 2006); de manera que las relaciones familiares y el contexto normativo cultural son inseparables. Así, las diferencias socioculturales se encuentren arraigadas en procesos comunicacionales basados en realidades particulares, fenómeno que, por lo demás, ha sido profusamente abordado por diversos autores desde distintas áreas de investigación lingüística en general (Briz, 2005; Charaudeau, 2012; Puga, 2012).

Las limitaciones del presente estudio están dadas por las características de la muestra, pues esta podría no ser representativa de los entornos rurales o más alejados del territorio nacional, donde es posible que la población posea otras características socioculturales distintivas. Asimismo, el estudio no ha contemplado la inclusión de la tercera alternativa educativa presente en el país - los establecimientos educativos particulares-, pues en caso de que se incluyera, podría otorgar mayor variabilidad a los resultados. En efecto, futuras investigaciones podrían abarcar una mayor diversidad poblacional a fin de comprobar el comportamiento de la escala en otros contextos sociales.

Comprender la comunicación familiar como un proceso de influencia mutua entre sus miembros que se lleva a cabo a lo largo de todo el ciclo vital del ser humano implica reconocer que se trata de un elemento esencial 
en el desarrollo y trayectoria evolutiva de cada persona. Por tanto, el estudio de la comunicación familiar facilita no solo la comprensión de la interacción del individuo en relación a la familia, sino, además, cómo es que la familia se relaciona con el medio social. Por esta razón, avanzar en el conocimiento acerca de los procesos interactivos que se producen al interior de la familia es un reto constante para diversas disciplinas y profesionales.

En esta línea, disponer de una escala de comunicación familiar válida en el contexto nacional chileno permitirá a los equipos multidisciplinarios que trabajan en distintos ámbitos relacionados con la prevención, promoción e intervención en el área familiar contar con un instrumento que oriente el quehacer profesional, y también facilitará el desarrollo de futuras investigaciones que posibiliten un mayor conocimiento de la temática, para así superar los vacíos empíricos actuales en el contexto nacional chileno y, del mismo modo, contribuir al desarrollo de la temática en América Latina.

\section{REFERENCIAS}

Arias, B. (2008). Desarrollo de un ejemplo de análisis factorial confirmatorio con LISREL, AMOS y SAS. En M.A Vergudo, M. Crespo, M. Badía \& B. Arias (Coords.), Metodología en la investigación sobre discapacidad. Introducción al uso de las ecuaciones estructurales (pp. 75-120). Valladolid: Publicaciones del INICO.

Banovcinova, A., \& Levicka, K. (2015). The impact of the financial income on the family communication. Revista Romaneasca Pentru Educatie Multidimensionala, 7(2), 35-46. doi: 10.18662/rrem/2015.0702.03.

Barcelata, B., \& Alvarez, I. (2005). Patrones de interacción familiar de madres y padres generadores de violencia y maltrato infantil. Acta Colombiana de Psicología, 13, 35-45.

Barnes, H. L., \& Olson, D. H. (1982). Parent-adolescent communication scale. En D. H. Olson, H. McCubbin, H. L. Barnes, A. Larsen, M. Muxen, \& M. Wilson (Eds.), Family inventories: Inventories used in a national survey of families across the family life cycle (pp. 51-63). St. Paul, MN: University of Minnesota.

Briz, A. (2005). Atenuación y cortesía verbal en la conversación coloquial: su tratamiento en la clase de ELE. Articulo presentado en Actas del Programa de Formación del Profesorado de Español como Lengua Extranjera, Munich.

Bronfenbrenner, U. (2005). Making human beings human: Bioecological perspectives on human development. Thousand Oaks, California: Sage.

Burns, M. E., \& Pearson, J. C. (2011). An exploration of family communication enviroment, everyday talk, and family satisfaction. Communication Studies, 62(2),171-185. doi: 10.1080/10510974.2010.523507.
Charaudeau, P. (2012). Problemas teóricos y metodológicos en los estudios de la oralidad aplicados a la cortesía: Aspectos lingüísticos, pragmáticos y discursivos. En J. Escamilla \& G. Henry (Eds.), Miradas multidisciplinarias a los fenómenos de cortesía y descortesía en el mundo hispánico. (1 ra. ed., pp. 13-32). Barranquilla: Universidad del AtlánticoCADIS.

Corsi, J. (1999). Violencia familiar: una mirada interdisciplinaria sobre un grave problema social. Buenos Aires: Paidós.

Estévez, E., Herrera, J., Martínez, B., \& Musitu, G. (2006). Aggresive and nonaggresive rejected students. An analysis of their differences. Psychology in the Schools, 43(3), 387400. doi: 10.1002/pits.20152.

Estévez, E., Musitu, G., \& Herrero, J. (2005). The influence of violent behavior and victimization at school on psychological distress: The role of parents and teachers. Adolescence, 40(157), 183-196.

Fitzpatrick, M. A., \& Ritchie, L. D. (1993). Communication theory and the family. En P. Boss, W. Doherty, R. LaRossa, W. Schumm, \& S. Steinmetz (Eds.), Sourcebook of family theories and methods: A contextual approach (pp. 565585). New York: Plenum.

Gallego, S. (2006). Comunicación familiar. Un mundo de construcciones simbólicas y relacionales. Manizales: Universidad de Caldas.

Galvin, K. M. (2015). The family communication tree: Deep roots, strong branches. En L. H. Turner \& R. West (Eds.), The Sage Handbook of Family Communication (pp. 1-8). Thousand Oaks, California: SAGE.

Girbau, M. D. (2002). Psicología de la comunicación. Madrid: Ariel.

Hooper, D., Coughlan, J., \& Mullen, M. (2008). Structural equation modelling: Guidelines for determining model fit. The Electronic Journal of Business Research Methods, 6(1), 53-60.

Isaza, L., \& Henao, G. (2011). Relaciones entre el clima social familiar y el desempeño en habilidades sociales en niños y niñas entre dos y tres años de edad. Acta Colombiana de Psicología, 14(1), 19-30.

Jiménez, T. I., Murgui, S., Estévez, E., \& Musitu, G. (2007). Comunicación familiar y comportamientos delictivos en adolescentes españoles: El doble rol mediador de la autoestima. Revista Latinoamericana de Psicología, 39(3), 473485.

Kerr, M., \& Stattin, H. (2000). What parent know, how they know it, and several forms of adolescent adjustment. Developmental Psychology, 36, 366-380. doi: 10.1037/00121649.36.3.366.

Kodali, S., Stametz, R., Clarke, D., Bengier, A., Sun, H., Layon, A. J., \& Darer, J. (2015). Implementing family communication pathway in neurosurgical patients in an intensive care unit. Palliative \& Supportive Care, 13(4), 961-967. doi:10.1017/s1478951514000650. 
Koerner, A., \& Fitzpatrick, M. A. (2004). Communication in intact families. En A. Vangelisti, Handbook of Family Communication (pp. 177-196). Mahwah, New Jersey: Lawrence Erlbaum Associates, Publishers.

Koerner, A., \& Fitzpatrick, M. A. (2002). Toward a theory family communication. Communication Theory, 12(1), 70-91. doi: 10.1111/j.1468-2885.2002.tb00260.x.

Lambert, S. F., \& Cashwell, C. S. (2003). Preteens talking to parents: Perceived communication and schoolbased aggression. The Family Journal: Counseling and Therapy for Couples and Families, 11, 1-7. doi: $10.1177 / 1066480703261953$.

Lecannelier, F., Pérez, J., Groissman, S., Gallardo, D., Bardet, A., Bascuñan, A., \& Rodriguez, J. (2014). Validación del inventario de conductas infantiles para niños entre 11/25 años (CBCL-11/2-5) en la ciudad de Santiago de Chile. Universitas Psychologica, 13(2), 491-500. doi: 10.11144/ Javeriana.UPSY13-2.vici.

Lecannelier, F., Varela, J., Rodríguez, J., Hoffmann, M., Flores, F., \& Ascania, L. (2011). Validación del cuestionario de maltrato entre iguales por abuso de poder (MIAP) para escolares. Revista Médica de Chile, 139(4), 474-479. doi: 10.4067/S0034-98872011000400009.

Leenen, C., Den Heijer, M., Van der Meer, C., Kuipers, E. J., Van Leerdam, M. E., \& Wagner, A. (2016). Genetic testing for Lynch syndrome: family communication and motivation. Familial Cancer, 15(1), 63-73. doi:10.1007/s10689015-9842-8.

Levin, K. A., Dallago, L., \& Currie, C. (2012). The association between adolescent life satisfaction, family structure, family affluence and gender differences in parent-child communication. Social Indicators Research, 106(2), 287-305. doi: 10.1007/s11205-011-9804-y.

López, V., Bilbao, M., Ascorra, P., Moya, I., \& Morales, M. (2014). Escala de clima escolar: Adaptación al español y validación en estudiantes chilenos. Universitas Psychologica, 13(3), 1111-1122. doi: 10.11144/Javeriana.UPSY13-3.ecea.

Luna, A. (2012). Funcionamiento familiar, conflictos con los padres y satisfacción con la vida de familia en adolescentes bachilleres. Acta Colombiana de Psicología, 15(1), 77-85.

Martínez, B., Musitu, G., Murgui, S., \& Amador, V. (2009). Conflicto marital, comunicación familiar y ajuste escolar en adolescentes. Revista Mexicana de Psicología, 26(1), 2740.

Mathiesen, M. E., Merino, J., Herrera, M. O., Castro, G., \& Rodríguez, C. (2011). Validación del funcionamiento de la Escala Ecers-r en algunas regiones de Chile. Estudios Pedagógicos (Valdivia), 37(2), 147-160. doi:10.4067/s071807052011000200009.

Moos, R.H., Moos, B.S., \& Trickett, E.J. (1987). Escalas de Clima Social. Madrid: TEA Ediciones.

Musitu, G., \& Cava, M.J. (2001). La familia y la educación. Barcelona: Octaedro.
Myers, M. F., Fernandes, S. L., Arduser, L., Hopper, J. L., \& Koehly, L. M. (2015). Talking about type 2 diabetes family communication from the perspective of at-risk relatives. Diabetes Educator, 41(6), 716-728. doi:10.1177/0145721715604367.

O'Toole, S., Benson, A., Lambert, V., Gallagher, P., Shahwan, A., \& Austin, J. K. (2015). Family communication in the context of pediatric epilepsy: A systematic review. Epilepsy y Behavior, 51, 225-239. doi:10.1016/j.yebeh.2015.06.043.

Parra, A., \& Oliva, A. (2002). Comunicación y conflicto familiar durante la adolescencia. Anales de Psicología, 18(2), 215-231.

Perrone, R., \& Nannini, M. (2000). Violencia y abusos sexuales en la familia. Buenos Aires: Paidós.

Presa, M. (2015). Estrés en la familia y funcionamiento familiar en un hospital psiquiátrico de México (Tesis de master), Universidad Pontificia Comillas, Madrid.

Puga, J. (2012). La observación participante en el estudio de la atenuación del castellano de Chile. En J. Escaramilla \& G. Henry (Eds.), Miradas multidisciplinarias a los fenómenos de cortesía y descortesía en el mundo hispánico (pp. 413450). Barranquilla: Universidad del Atlántico- CADIS.

Puschel, K., Repetto, P., Olga Solar, M., Soto, G., \& Gonzalez, K. (2012). Design and validation of an instrument to assess families at risk for health problems. Revista Médica de Chile, 140(4), 417-425. doi: 10.4067/S003498872012000400001.

Pérez, M., \& Aguilar, J. (2009). Relaciones del conflicto padresadolescentes con la flexibilidad familiar, comunicación y satisfacción marital. Psicología y Salud, 19(1), 111-120.

Quintana, T., \& Muñoz, G. (2010). Validación y adaptación del cuestionario de temperamento y carácter, JTCI de R. Cloninger, versión niños y padres, a escolares chilenos entre 8 y 13 años de edad. Terapia Psicológica, 28(1), 37-43. doi: 10.4067/S0718-48082010000100004.

Retamales, J., Behn, V., \& Merino, J. (2004). Funcionamiento familiar de trabajadores-jefes de hogar en sistema de turnos de una empresa de Talcahuano, Chile, año 2003. Ciencia y Enfermería, X(1), 23-30. doi: 10.4067/S071795532004000100004.

Rivadeneira, J. (2013). El Funcionamiento familiar, los estilos parentales y el estímulo al desarrollo de la teoría de la mente: Efectos en criaturas sordas y oyentes (Tesis doctoral), Barcelona: Universidad Autónoma de Barcelona.

Rivero, N., \& Martínez-Pampliega, A. (2010). Adaptación cultural del Instrumento "Patrones de Comunicación FamiliarR". European Journal of Education and Psychology, 3(1), 141-153. doi: 10.1989/ejep.v3i1.52.

Rudy, J., Dworkin, J., Walker, S., \& Doty, J. (2015). Parents' use of information and communications technologies for family communication: differences by age of children. Information, Communication y Society, 18(1), 78-93. doi: 10.1080/1369118X.2014.934390. 
Ruiz, M., Pardo, A., \& San Martín, R. (2010). Modelos de ecuaciones estructurales. Papeles del Psicólogo, 31(1), 34-45.

Saldivia, S., Vicente, B., Valdivia, M., \& Melipillán, R. (2013). Validación de la entrevista diagnóstica estructurada DISCIV para la identificación de trastornos psiquiátricos en niños y adolescentes en Chile. Revista Chilena de Neuro-Psiquiatría, 51(1), 70-78. doi:10.4067/s0717-92272013000100009.

Santander, S., Zubarew, T., Santelices, L., Argollo, P., Cerda, J., \& Bórquez, M. (2008). Influencia de la familia como factor protector de conductas de riesgo en escolares chilenos. Revista Médica de Chile, 136(3), 317-324. doi. 10.4067/ S0034-98872008000300006.

Sanz, M., Iraurgi, I., \& Martínez-Pampliega, A. (2002). Evaluación del funcionamiento familiar en toxicomanías: Adaptación española y características de adecuación métrica del FAP-FACES IV. En I. Iraurgi \& F. González-Saiz (Eds.), Instrumentos de evaluación en drogodependencias (pp. 403-434). Madrid: Aula Médica.

Segrin, C., \& Flora, J. (2011). Family communication (Second ed.). New York: Routledge.

Smith, K. M., Freeman, P. A., \& Zabriskie, R. (2009). An examination of family communication within the core and balance model of family leisure functioning. Family Relations, 58(1), 79-90. doi: 10.1111/j.1741-3729.2008.00536.x.

Stage, J., King, F., Nora, A., \& Barlow, E. (2006). Reporting structural equation modeling and confirmatory factor analysis results: A review. The Journal of Education Research, 99(6), 323-337. doi: 10.3200/JOER.99.6.323-338.
Tesson, G., \& Youniss, J. (1995). Micro-sociology and psychological development: A sociological interpretation of Piaget's theory. En A. M. Ambert (Ed.), Sociological studies of children (Vol. 7, pp. 101-126). Greenwich, CT: JAI.

Wang, M. P., Chu, J. T. W., Viswanath, K., Wan, A., Lam, T. H., \& Chan, S. S. (2015). Using information and communication technologies for family communication and its association with family well-being in Hong Kong: FAMILY Project. Journal of Medical Internet Research, 17(8), e207. doi: 10.2196/jmir.4722.

Webb, L. M., \& Dickson, F. C. (2012). Effective family communication for coping with crises. En F. C. Dickson \& L. M. Webb (Eds.), Communication for families in crisis: Theories, methods, strategies (pp. 1-26). New York: Peter Lang.

Wolf, E., Harrington, K., Clark, S., \& Miller, M. (2013). Sample size requirements for structural equation models: An evaluation of power, bias, and solution propriety. Educational and Psychological Measurement, 76(6), 931-934. doi: 10.1177/0013164413495237.

Zicavo, N., Palma, C., \& Garrido, G. (2012). Adaptación y validación del Faces-20-ESP: Re-conociendo el funcionamiento familiar en Chillán, Chile. Revista Latinoamericana de Ciencias Sociales, Niñez y Juventud, 10(1), 219-234. 